\title{
Fundamentos lógicos de las ontologías Web
}

\section{1}

\author{
Elsa E. Barber, Silvia Pisano, Sandra Romagnoli, Gabriela de Pedro, \\ Carolina Gregui y Nancy Blanco \\ Universidad de Buenos Aires. Facultad de Filosofía y Letras. Instituto de Investigaciones Bibliotecológicas. Ciudad Autónoma de \\ Buenos Aires, Argentina \\ elsabarber.eb@gmail.com / https://orcid.org/oooo-0003-2970-1356 | silvialuisapisano@gmail.com / \\ https://orcid.org/oooo-0003-1408-907X | sandraer@fibertel.com.ar/ https://orcid.org/oooo-0003-4479-3172 I \\ gabdp@yahoo.com / https://orcid.org/oooo-ooo1-9409-2644 | cgregui@hotmail.com / https://orcid.org/oooo-ooo3-2916-3543 | \\ nancybl@filo.uba.ar / https://orcid.org/oooo-0oo2-4218-6187
}

\begin{abstract}
Resumen
El artículo define las ontologías en el ámbito de la Web semántica, donde se las describe como artefactos representacionales, dispositivos jerárquicos constituidos por entidades y relaciones, y se las tipifica de acuerdo con su grado de generalidad y su funcionalidad. Se destacan los principios básicos que las rigen y las buenas prácticas a tener en cuenta a nivel lógico para su diseño e implementación de acuerdo con la Basic Formal Ontology (BFO), orientada a sustentar las cuestiones lógicas de todo tipo de ontologías. Esquematiza las clases que incluye BFO, sus relaciones y principales axiomas. Subraya el impacto de la expresividad de los lenguajes utilizados al diseñar ontologías en cuanto a la resolución de los aspectos formales que influyen en la capacidad de éstas para generar inferencias automáticamente.
\end{abstract}

\section{Logical foundations of Web ontologies}

\begin{abstract}
The article defines the ontologies in the Semantic Web, where they are described as representational artifacts, hierarchical devices constituted by entities and relationships, and are typified according to their degree of generality and functionality. It highlights the basic principles that govern them and good practices to be taken into account at the logical level for their design and implementation in accordance with the Basic Formal Ontology (BFO) oriented to support the logical issues of all types of ontologies. It defines the classes that BFO includes, its relationships and main axioms. It emphasizes the impact of the expressiveness of the languages used when designing ontologies in terms of the resolution of formal aspects that influence the ability of these to generate inferences automatically.
\end{abstract}

Artículo recibido: 10-04-2019. Aceptado: 18-09-2019.

\section{Palabras clave}

Lógica

Diseño

Ontología

Web

BFO

Basic Formal Ontology

\author{
Keywords \\ Logic \\ Design \\ Ontology \\ Web \\ BFO \\ Basic Formal Ontology
}




\section{Introducción}

Desde la perspectiva filosófica, ontología es la teoría de lo que existe, el estudio de lo que es, de los diversos objetos, de sus estructuras, propiedades, eventos, procesos y relaciones en la realidad (Guarino, Oberle y Staab, 2009). Su alcance es universal, se orienta hacia el análisis de las características de la realidad comunes a todos los dominios, unidad / pluralidad, causa / efecto, espacio / tiempo, identidad / diferencia, parte / todo; trata de identificar y clasificar las distintas entidades (Arp, Smith y Spear, 2015). En el ámbito de la Ciencia de la Computación, para un sistema lo que "existe" solo es aquello que puede ser "representado" (Gruber, 1993; Guarino, Oberle y Staab, 2009), por lo tanto, se la define como “...a representational artifact, comprising a taxonomy as proper part, whose representations are intended to designate some combination of universals, defined classes, and certain relations between them." (Smith, Kusnierczyk, Schober y Ceusters, 2006, p.61), o como:

... an engineering artifact, constituted by a specific vocabulary used to describe a certain reality, plus a set of explicit assumptions regarding the intended meaning of the vocabulary words. This set of assumptions has usually the form of a firstorder logical theory, where vocabulary words appear as unary or binary predicate names, respectively called concepts and relations. In the simplest case, an ontology describes a hierarchy of concepts related by subsumption relationships; in more sophisticated cases, suitable axioms are added in order to express other relationships between concepts and to constrain their intended interpretation. (Guarino, 1998, p. 4)

Es, entonces, algo deliberadamente seleccionado o diseñado por los seres humanos con un propósito particular (i.e., un artefacto). En el caso de la ontología dicho propósito es la representación, definida como una entidad que refiere a otra u otras entidades externas a ella. Las entidades, por lo tanto, comprenden todo lo que existe, tanto objetos, procesos y cualidades como, por ejemplo, representaciones, imágenes, observaciones y documentos. Si se tienen en cuenta tres niveles de entidades, la realidad; las representaciones cognitivas de la realidad y los artefactos representacionales (la objetivación textual o gráfica de las representaciones cognitivas), las ontologías se incluyen en este último nivel (Smith, Kusnierczyk, Schober y Ceusters, 2006). Cabe destacar que las ontologías se plantean describir y representar adecuadamente aquellas estructuras de la realidad que se corresponden con los términos usados por los científicos; no se hallan orientadas hacia los conceptos, al término en sí mismo, sino hacia la realidad (Arp, Smith y Spear, 2015).

Las ontologías se organizan como dispositivos jerárquicos estructurados en grafos constituidos por nodos (representan tipos, universales o clases, entidades pertenecientes a la realidad) y aristas (representan relaciones is_a, equivalentes a "es un subtipo de" que vinculan a los universales). Cuentan con un nodo "raíz" conectado a todos los otros nodos mediante un único recorrido, de manera que todos los nodos por debajo del nodo inicial tienen solo un nodo padre. Es decir, que la columna vertebral de una ontología se halla constituida por una generalización / especialización jerárquica de conceptos, a la manera de una taxonomía (Guarino, Oberle y Staab, 2009).

Las ontologías pueden tipificarse de acuerdo con su grado de generalidad, en genéricas, de dominio o de aplicación (Guarino, 1998; Vitturini y Fillottrani, 2012); según su estilo de integración a un sistema semántico, en sistema de ontología única para todas las aplicaciones, de múltiples ontologías o híbrido, más flexible, que combina los anteriores para establecer una ontología de dominio (Wache et al., 2001). Para el propósito de este trabajo interesa distinguir entre las ontologías formales o genéricas y las ontologías materiales o de dominio. Las primeras contienen solo términos muy 
generales que se aplican en todas las disciplinas, universales neutros representados en múltiples ontologías, categorías, cuyas instancias se encuentran en cualquier dominio de la realidad.

Las ontologías materiales, en cambio, se diseñan para un dominio específico, contienen términos que se aplican solo a un número acotado de disciplinas, se hallan constituidas por representaciones de universales materiales, no formales, instanciados en un dominio específico de la realidad. De todas maneras, ambas se encuentran conformadas por una taxonomía estructurada jerárquicamente por la relación is_a, además de otras relaciones (partitivas, participativas, de adyacencia, de precedencia, etc.), así como por definiciones y axiomas que indican como deben interpretarse los términos y las relaciones que la integran (Arp, Smith y Spear, 2015):

Definitions are perhaps the most important component of ontologies, since it is through definitions that an ontology draws its ability to support consistent use across multiple communities and disciplines, and to support computational reasoning. Definitions also constrain the organization of the ontology. (p. 36)

Para Aristóteles, todas las definiciones requieren un punto de partida (genus, padre), en relación con el cual un término (especie, hijo) puede ser definido. En una ontología material o de dominio, ese punto de partida se encuentra en su nodo raíz. Dicho nodo, así como otros términos en el nivel superior de la ontología si fuera necesario, deben expresar una categoría o universal neutral, válida para todos los dominios. Las ontologías formales, proporcionan, entonces, los términos que garantizan homogeneidad semántica e interoperabilidad a fin de facilitar la comprensión de los seres humanos y la capacidad de análisis de las computadoras a través de los motores de razonamiento.

Cabe, además, mencionar las ontologías de aplicación que se crean para realizar una tarea puntual, a diferencia de las de referencia que, por el contrario, otorgan una representación canónica de las entidades pertenecientes a un dominio, es decir, del conocimiento consolidado. Por ese motivo, sus términos son reutilizados cuando se encara el diseño de una ontología de aplicación (Shaw, Detwiler, Brinkley y Suciu, 2008).

Las cuestiones relacionadas con la integración e interoperabilidad de las ontologías han sido motivo permanente de indagación por parte de los investigadores interesados en el desarrollo de estas herramientas como artefacto computacional apto para soportar la interrogación y la recuperación de información científica en el ámbito de la web semántica. En el marco de esta problemática, un manejo adecuado del nivel lógico resulta fundamental para dar cohesión y coherencia a las ontologías a fin de que funcionen adecuadamente. Diversos autores consideran que las ontologías formales, constituyen el eje rector capaz de proporcionar la estructura de base que garantice la consistencia de estas en ese nivel (Arp y Smith, 2008, 2011; Arp, Smith y Spear, 2015; Guarino, 1995, 1998; Guarino y Welty, 2000, 2009; Guarino, Oberle y Staab, 2009; Mascardi, Cordì, y Rosso, 2007; Poli, Healy y Kameas, 2010; Smith et al., 2005; Smith, Kusnierczyk, Schober y Ceusters, 2006; Vitturini y Fillottrani, 2012).

Mascardi, Cordì, y Rosso, 2007, describen y comparan siete ontologías de nivel superior; entre ellas, la Basic Formal Ontology (BFO; http://basic-formal-ontology.org/), ha sido diseñada, según sus creadores, para la recuperación, análisis e integración de información en dominios científicos. BFO, resulta de interés porque:

" como ontología general, sistematiza las entidades y relaciones que permiten estructurar adecuadamente el nivel lógico de una ontología en cualquier especialidad, 
» tanto sus bases teóricas como sus aspectos técnicos han sido documentados y analizados en numerosas publicaciones (véase http://basic-formal-ontology. org/publications.html),

» ha sido adoptada por más de 250 iniciativas de diseño de ontologías a escala mundial y posee un foro activo de discusión en el que intervienen sus desarrolladores y su comunidad de usuarios,

» ha sido propuesta como estándar ISO (ISO/IEC 21838: Top-Level Ontologies),

" ha sido utilizada por la Information Artifact Ontology (IAO), ontología de entidades de información, que constituyen un subtipo (continuos genéricamente dependientes) dentro de las entidades de BFO,

» dispone de implementación OWL, a la que es possible acceder en forma gratuita.

Por ese motivo, este trabajo tiene por objetivo presentar, sobre la base de los aportes realizados por sus creadores, las características esenciales de la Basic Formal Ontolo$g y$, en cuanto ontología superior con capacidad para resolver el nivel lógico de una ontología material o de dominio, a fin de proporcionar a los especialistas en Ciencia de la Información el marco teórico capaz de respaldar el diseño de distintos tipos de ontologías.

En ese contexto, para Arp, Smith y Spear (2015) es importante señalar las características de los universales y de los particulares, así como de las clases con respecto a ellos (Tabla 1). Los universales son repetibles, pueden instanciarse en más de un objeto y más de una ocasión, son propiedades de la realidad, independientes de nuestra mente, que existen solo instanciados; los universales dependen para su existencia de los particulares. Estos últimos, en cambio, son irrepetibles, solo existen en un espacio y un tiempo dados, instancian a los universales, pero no pueden ser instanciados. La máxima colección de particulares agrupados bajo un término dado conforma una clase denominada "natural" y define la extensión del universal correspondiente.

\begin{tabular}{|c|c|c|c|}
\hline \multicolumn{4}{|c|}{ Ontologías } \\
\hline Universales & $\begin{array}{l}\text { Entidades } \\
\text { representadas por } \\
\text { términos generales } \\
\text { usados en la } \\
\text { formulación de } \\
\text { leyes científicas. } \\
\text { Se organizan } \\
\text { jerárquicamente }\end{array}$ & $\begin{array}{l}\text { Son repetibles } \\
\text { Pueden } \\
\text { instanciarse en } \\
\text { más de un objeto } \\
\text { y en más de una } \\
\text { ocasión }\end{array}$ & $\begin{array}{l}\text { Son características } \\
\text { de la realidad } \\
\text { Existen solo } \\
\text { instanciados } \\
\text { Dependen para su } \\
\text { existencia de los } \\
\text { particulares }\end{array}$ \\
\hline Particulares & $\begin{array}{l}\text { Individuos reales } \\
\text { de cualquier tipo }\end{array}$ & $\begin{array}{l}\text { Son irrepetibles } \\
\text { Solo existen en } \\
\text { un espacio y un } \\
\text { tiempo dados }\end{array}$ & $\begin{array}{l}\text { Instancian a los } \\
\text { universales, pero } \\
\text { no pueden ser } \\
\text { instanciados }\end{array}$ \\
\hline Clases Naturales & $\begin{array}{l}\text { Máxima colección } \\
\text { de particulares } \\
\text { agrupados bajo } \\
\text { un término } \\
\text { general que define } \\
\text { la extensión } \\
\text { del universal } \\
\text { correspondiente }\end{array}$ & & \\
\hline
\end{tabular}

Tabla 1. Ontologías 
Existen, además, para estos autores, las clases "definidas" (Tabla 2). Es posible distinguir al menos dos familias de clases definidas:

1. Clases definidas por términos generales con la capacidad de abreviar combinaciones lógicas de términos que denotan universales. Se dividen en dos grupos:

a) Definidas por selección: por ejemplo, varones con ojos azules; tales clases son subclases dentro de la extensión de un universal dado, se definen a través de la conjunción lógica;

b) Definidas por combinación: comprenden miembros que instancian dos o más universales que no se superponen. Son uniones de las extensiones de los universales involucrados, se definen por medio de la disyunción lógica (por ejemplo, empleado o asalariado);

2. Clases definidas por términos generales que abrevian combinaciones lógicas de términos que denotan universales con términos que denotan particulares, por ejemplo, hombres que viven actualmente en el norte de Argentina, músicos nacidos después de 1990.

\begin{tabular}{|c|c|c|c|}
\hline \multicolumn{4}{|c|}{ Clases definidas } \\
\hline $\begin{array}{r}\text { Clases que } \\
\text { denotan } \\
\text { universales }\end{array}$ & $\begin{array}{l}\text { Definidas por términos } \\
\text { generales con la } \\
\text { capacidad de abreviar } \\
\text { combinaciones lógicas } \\
\text { que denotan universales }\end{array}$ & $\begin{array}{l}\text { Definidas por } \\
\text { selección }\end{array}$ & $\begin{array}{l}\text { Conjunción lógica } \\
\text { Disyunción lógica }\end{array}$ \\
\hline $\begin{array}{r}\text { Clases que } \\
\text { denotan } \\
\text { universales y } \\
\text { particulares }\end{array}$ & $\begin{array}{l}\text { Definidas por términos } \\
\text { generales que abrevian } \\
\text { combinaciones lógicas } \\
\text { de términos que } \\
\text { denotan universales con } \\
\text { términos que denotan } \\
\text { particulares }\end{array}$ & & \\
\hline
\end{tabular}

Tabla 2. Clases definidas

Fuente de los datos: Arp, Smith y Spear (2015)

Hay cuatro principios básicos a seguir al elaborar una ontología: realismo, perspectivismo, falibilidad y adecuación. A ellos se suman algunos principios adicionales (Arp, Smith y Spear, 2015):

»Deben ser reutilizables

"El proceso de diseño debe

- equilibrar utilidad y realismo

- ser abierto

- reunir en primer lugar los términos más generales y fáciles de definir dentro del dominio, para luego representar las entidades más complejas

Según Arp, Smith y Spear (2015), los pasos a seguir para diseñar una ontología de dominio son los siguientes:

1. Demarcar el alcance; la materia y el nivel de granularidad de la realidad a representar

2. Reunir información; identificar los términos generales más usados en el dominio, verificar su existencia en ontologías de referencia, eliminar las redundancias 
3. Ordenar los términos resultantes jerárquicamente, de lo general a lo específico

4. Normalizar el resultado para asegurar:

a. Coherencia lógica, filosófica y científica

b. Coherencia y compatibilidad con ontologías vinculadas

c. Comprensión por parte de los seres humanos a través de definiciones consistentes

5. Formalizar el artefacto representacional normalizado en un lenguaje de computación de tal manera que el resultado pueda implementarse en entorno automatizado

De acuerdo con estos autores, las buenas prácticas indican, además, que:

\section{con respecto a la terminología}

" solo se deben representar aquellas clases para las que existen instancias, para las que hay evidencia de que se poseen miembros

»los nombres y frases comunes preferidos en el dominio forman el primer esbozo sobre el que se construye la terminología

» para los tipos de entidades más importantes, se usan los términos preferidos por los grupos de científicos más influyentes, si es necesario, se consensuan dichos términos y se acuerda un compromiso de uso

" se asegura el significado unívoco de los términos adoptados, aunque múltiples términos pueden referir a uno preferido con el cual han sido asociados

» se garantiza la unicidad de las expresiones relacionales

» se identifican áreas de solapamiento disciplinar donde el uso de los términos no es consistente, se buscan sinónimos adecuados para reemplazarlos

" si no es posible reemplazarlos se desarrollan múltiples ontologías o ramas diversas en una única ontología y se conectan los términos en cuestión mediante definiciones, axiomas y relaciones apropiadas

" es conveniente hacer uso, cuanto sea posible, de los recursos terminológicos ya existentes en otras ontologías

» es primordial usar nombres y frases comunes que refieran a un universal o a una clase definida, en singular para que los razonadores de las aplicaciones trabajen correctamente

" los nombres comunes que refieren a universales o clases, se registran en letra itálica minúscula, las letras mayúsculas se usan en inglés para indicar nombres propios que corresponden a instancias

» algunos programas editores de ontologías exigen el uso de guion bajo, comillas - CamelCase (uso de mayúsculas en una posición distinta de la inicial) para normalizar los nombres constituidos por frases; es importante aplicar con consistencia la convención a la que se adhiera

» se evita el uso de acrónimos y abreviaturas

" cada término, para satisfacer los requerimientos computacionales, se asocia con un único identificador alfanumérico

» se usan nombres que pueden cuantificarse, se recomienda transformar los nombres no cuantificables mediante la inserción de un operador apropiado al inicio, por ejemplo, "porción de"

» no se crean términos para los universales a través de combinaciones lógicas, los términos negativos son evitados, los universales son en todos los casos positivos

" se evita la creación de entradas que constituyen el complemento de una clase (el complemento contiene todas las entidades que no pertenecen a la clase)

" se distingue lo general de lo particular. Cuando es necesario representar individuos, estos se incluyen en un artefacto separado, por ejemplo, T-box 
(Terminología) y A-box (Aserciones); ambos artefactos se combinan, luego, y forman la "base de conocimiento"

con relación a las definiciones

》 se proveen definiciones para todos los términos, es decir, se menciona el conjunto de condiciones necesarias y suficientes para describir el término; al mencionar las condiciones se usan términos más fáciles de comprender y más simples desde el punto de vista lógico que el término a definir

" las condiciones establecidas son conjuntamente satisfactorias

" se mencionan características esenciales (aquellos elementos que todas las instancias del universal deben poseer, sin los cuales una cosa deja de ser el tipo de cosa que es)

» se usan definiciones aristotélicas, $E$ = def. un G que Ds, donde $\mathrm{G}$ (género) es el orden inmediatamente superior de $\mathrm{E}$ (especie) en la ontología y $\mathrm{D}$ (diferencia) indica la distinción que justifica la existencia de la especie dentro del género; de esta manera se enmarca el término definido dentro de la jerarquía is_a (la taxonomía provee el elemento inicial para formular la definición). Es preferible que los términos usados para manifestar las diferencias se hayan extraído de alguna ontología donde ellos mismos son definidos

" cada definición, cuando se descompone, refiere al nodo raíz de la ontología a la que pertenece, así se elimina automáticamente la circularidad de las definiciones, el autor de la definición conoce siempre cuál es su punto de partida; es necesario que todos los nodos en el grafo se vinculen al nodo raíz mediante una única cadena de relaciones is_a

» se recurre a una ontología de nivel más alto para definir el término raíz, o se declara a este término como primitivo (los términos primitivos no son definidos pero su significado puede aclararse en una nota)

» se recomienda definir los términos desde los niveles superiores de la ontología hacia los niveles inferiores

" las definiciones son sustituibles por los términos definidos sin un cambio en el significado, ello es importante para preservar el criterio de verdad a través de los procesos de inferencia automática

en cuanto al rol de la taxonomía

» todas las ontologías se estructuran en torno a la columna vertebral constituida por la jerarquía is_a

» esta cualidad se testea mediante la validación de la afirmación $\mathrm{A}$ is_a $\mathrm{B}$, donde cada instancia de $A$ es una instancia de $B$, cuando $A$ y $B$ refieren a universales o a clases definidas

" asegura una sola herencia para cada término; como ya se ha dicho, ello garantiza una única cadena de relaciones padre/hijo. Se favorece así, la elaboración de definiciones de acuerdo con el criterio aristotélico y la capacidad del razonador automático para efectuar inferencias correctas

» la adecuada construcción de la taxonomía facilita la combinación automática de diversas ontologías cuando ello se requiere

" se sostiene una visión abierta del mundo (open-world assumption, OWA) que asume la posibilidad de extensión y corrección constantes de la ontología, ya que su objetivo último consiste en dar soporte al trabajo de los científicos de clasificar particulares

" se adhiere, a la regla de la objetividad, es decir, a describir lo que existe en realidad y no lo que se conoce sobre lo que existe en realidad 


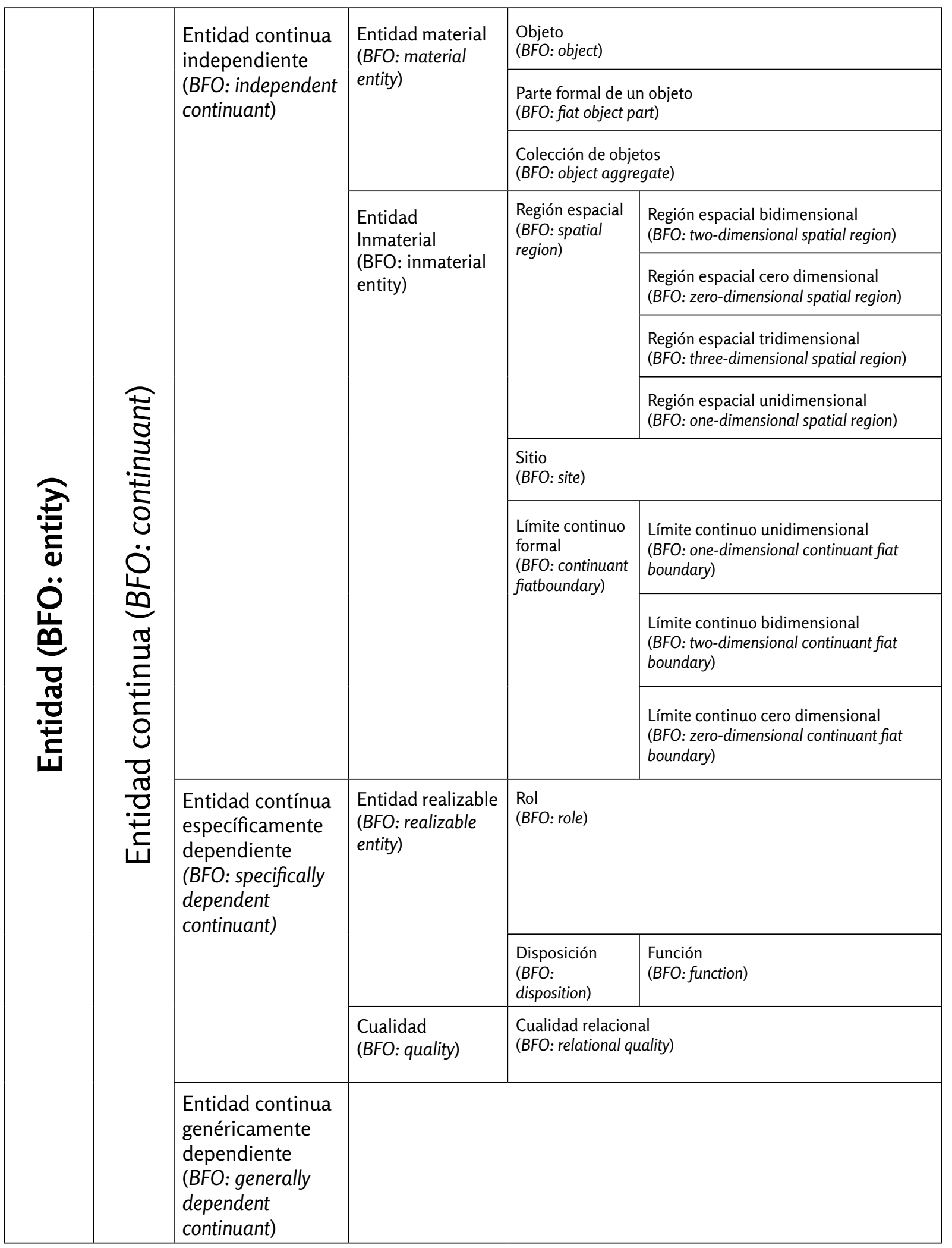

Tabla 3a. Entidades contínuas 


\begin{tabular}{|c|c|c|c|}
\hline \multirow{6}{*}{ 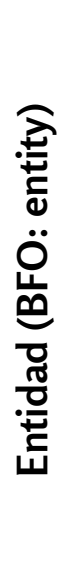 } & \multirow{6}{*}{ 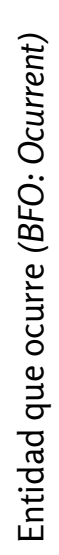 } & \multirow[t]{2}{*}{ Proceso (BFO: process) } & Historia (BFO: history) \\
\hline & & & Perfil del proceso (BFO: process profile) \\
\hline & & $\begin{array}{l}\text { Límite del proceso } \\
\text { (BFO: process boundary) }\end{array}$ & \\
\hline & & $\begin{array}{l}\text { Región temporal } \\
\text { (BFO: temporal region) }\end{array}$ & Región temporal unidimensional (BFO: one-dimensional temporal region) \\
\hline & & & Región temporal cero dimensional (BFO: zero-dimensional temporal region) \\
\hline & & $\begin{array}{l}\text { Región espaciotemporal } \\
\text { (BFO: spatiotemporal region) }\end{array}$ & \\
\hline
\end{tabular}

Tabla 3b. Entidades que ocurren

Fuente de los datos: Arp, Smith y Spear (2015)

La Basic Formal Ontology (BFO) considera las categorías básicas de la realidad, aquellas de nivel superior, comunes a todas las disciplinas. Contribuye a determinar dónde deben posicionarse las entidades dentro de la ontología, a determinar su definición y sus relaciones. El objetivo que subyace es la interoperabilidad de datos y sistemas de información sin la intervención de los seres humanos, como mecanismo para contribuir a la creación de nuevo conocimiento y actualizar automáticamente la representación del conocimiento alcanzado, así como las particularidades e idiosincrasias de comunidades y tecnologías de investigación (Smith, 1998, 2015; Arp, Smith y Spear, 2015).

\section{Clases de la Basic Formal Ontology (BFO)}

Arp, Smith y Spear (2015), han incorporado 35 clases estructuradas a la BFO, sobre la base de la siguiente taxonomía (Tabla 3):

Las clases incluidas en BFO han sido definidas conforme al criterio aristotélico (Tabla 4):

\begin{tabular}{|l|l|}
\hline \multicolumn{1}{|c|}{ Clases } & \multicolumn{1}{c|}{ Definición } \\
\hline Entidad continua & $\begin{array}{l}\text { Es una entidad que persiste, perdura o continúa existiendo a través del tiempo mientras mantiene su } \\
\text { identidad }\end{array}$ \\
\hline $\begin{array}{l}\text { Entidad continua } \\
\text { Independiente }\end{array}$ & $\begin{array}{l}\text { Es una entidad continua portadora de cualidades. Dichas cualidades son inherentes a la entidad } \\
\text { La inherencia puede definirse como una dependencia unilateral que se obtiene entre las cualidades, las } \\
\text { disposiciones y los roles, por un lado, y las entidades continuas independientes, por el otro } \\
\text { La dependencia implica que la entidad dependiente es secundaria (ha disminuido la especificidad) en } \\
\text { relación con la entidad continua independiente que es su portador } \\
\text { Una entidad continua independiente, dado que es una entidad en la cual otras entidades (como las } \\
\text { cualidades) se encuentran, no puede en sí misma ser inherente a nada }\end{array}$ \\
\hline Entidad material & $\begin{array}{l}\text { Es una entidad continua independiente que tiene una porción de materia como parte. Se extiende en el } \\
\text { espacio tridimensional y existe durante un intervalo de tiempo }\end{array}$ \\
\hline
\end{tabular}




\begin{tabular}{|c|c|}
\hline Objeto & $\begin{array}{l}\text { Es una entidad continua independiente material, tridimensional, indivisible e interconectada } \\
\text { Los objetos son la base sobre la que depende la existencia de las entidades continuas dependientes y las } \\
\text { entidades que ocurren } \\
\text { Un objeto es una entidad que puede existir y ser lo que es independientemente de qué otros objetos } \\
\text { existan }\end{array}$ \\
\hline $\begin{array}{l}\text { Parte formal de un } \\
\text { objeto }\end{array}$ & $\begin{array}{l}\text { Es una parte de un objeto más extenso } \\
\text { Carece de discontinuidad física con respecto al objeto al que pertenece (por ejemplo, la parte superior } \\
\text { del torso de una persona) } \\
\text { No incluye las partes de objetos que constituyen objetos en sí mismas y poseen límites físicos propios }\end{array}$ \\
\hline Colección de objetos & $\begin{array}{l}\text { Es una entidad material que se compone de un conjunto de objetos } \\
\text { Sus partes se hallan constituidas exactamente por los objetos que forman ese conjunto } \\
\text { Puede ganar y perder partes sin modificar su composición numérica (por ejemplo, un equipo deportivo) }\end{array}$ \\
\hline Entidad inmaterial & $\begin{array}{l}\text { Es una entidad continua independiente cuyas partes se hallan constituidas por entidades no materiales } \\
\text { Puede ser de tres, dos, una o ninguna dimensión }\end{array}$ \\
\hline Región espacial & $\begin{array}{l}\text { Es una entidad continua independiente inmaterial que constituye una parte de la totalidad del espacio } \\
\text { existente (por ejemplo, las personas ocupamos una región espacial tridimensional) } \\
\text { Existe independientemente de las entidades materiales y, por lo tanto, no cambia } \\
\text { Puede especificarse solo en relación con un marco de referencia euclídeo, donde cada punto se identifica } \\
\text { mediante las coordenadas de su vector de posición } \mathrm{A}(\mathrm{x}, \mathrm{y})\end{array}$ \\
\hline Sitio & $\begin{array}{l}\text { Es una entidad inmaterial en la cual los objetos son o pueden ser contenidos } \\
\text { Existe porque hay alguna entidad material en relación con la cual puede ser definido } \\
\text { Puede cambiar de ubicación, forma y medida (por ejemplo, los límites de un país, los orificios nasales, la } \\
\text { bodega de un barco) } \\
\text { Todo sitio coincide en algún momento con una región espacial pero no es idéntico a ella porque } \\
\text { depende de los límites de la estructura que lo rodea, es decir, a diferencia de la región espacial, es } \\
\text { ontológicamente dependiente de su contenedor }\end{array}$ \\
\hline Límite continuo formal & $\begin{array}{l}\text { Es una entidad inmaterial de cero, una o dos dimensiones que no incluye una región espacial como parte } \\
\text { Intuitivamente es el límite o contorno de alguna entidad material (por ejemplo, la superficie de la tierra), } \\
\text { pero también puede ser el límite de una entidad inmaterial (por ejemplo, de la porción de espacio aéreo } \\
\text { en la que le es permitido volar a una aeronave) }\end{array}$ \\
\hline $\begin{array}{l}\text { Entidad continua } \\
\text { específicamente } \\
\text { dependiente }\end{array}$ & $\begin{array}{l}\text { Es una entidad continua que depende de alguna entidad continua independiente específica para existir, } \\
\text { que es su portadora } \\
\text { Una entidad continua específicamente dependiente no puede migrar de un portador a otro }\end{array}$ \\
\hline Entidad realizable & $\begin{array}{l}\text { Es una entidad continua específicamente dependiente inherente a una o más entidades continuas } \\
\text { independientes } \\
\text { Tiene al menos una entidad continua independiente como portadora } \\
\text { Sus instancias pueden manifestarse, actualizarse y ejecutarse en procesos asociados con tipos } \\
\text { específicos de correlaciones en los que el portador participa o pueden permanecer latentes }\end{array}$ \\
\hline Rol & $\begin{array}{l}\text { Es una entidad realizable opcional cuya manifestación implica algún resultado o final que no es esencial } \\
\text { al portador en virtud de su estructura física, pero implica un cambio al cesar } \\
\text { Es el papel que juega una instancia de una entidad continua porque dicha entidad se halla inmersa en un } \\
\text { conjunto de circunstancias naturales, sociales o institucionales especiales } \\
\text { Como las cualidades, no puede migrar de un portador a otro }\end{array}$ \\
\hline Disposición & $\begin{array}{l}\text { Es una entidad continua dependiente realizable cuando se producen los disparadores o detonantes } \\
\text { adecuados, es decir, causa un proceso determinado en el continuo independiente de la que es inherente } \\
\text { la disposición en cuestión } \\
\text { Dicho proceso ocurre como resultado de la estructura física del objeto o continuo independiente }\end{array}$ \\
\hline Función & $\begin{array}{l}\text { Es una clase especial de disposición que existe en virtud de las características físicas intrínsecas del } \\
\text { portador, a través de la selección natural en el caso de entidades biológicas o de un diseño intencional en } \\
\text { el caso de los artefactos } \\
\text { Una función es una entidad continua dependiente realizable } \\
\text { Tiene un portador, que es una entidad continua independiente } \\
\text { Sus instancias tienen realizaciones } \\
\text { Cada realización es un proceso en el que el portador es participante en virtud de la constitución física } \\
\text { que posee a partir de su existencia }\end{array}$ \\
\hline Cualidad & $\begin{array}{l}\text { Se hereda plenamente realizada y manifestada en la entidad } \\
\text { Puede heredarse en uno o múltiples continuos independientes } \\
\text { Se ordena desde la más general (determinable, esencial, constante en la arquitectura del portador) hasta } \\
\text { la más específica (determinada, variable en el tiempo) }\end{array}$ \\
\hline
\end{tabular}




\begin{tabular}{|c|c|}
\hline Cualidad relacional & $\begin{array}{l}\text { Tiene una pluralidad de entidades continuas independientes como portadoras. Representa todas las } \\
\text { relaciones que se dan entre personas (por ejemplo, "ser padre de") }\end{array}$ \\
\hline $\begin{array}{l}\text { Entidad continua } \\
\text { genéricamente } \\
\text { dependiente }\end{array}$ & $\begin{array}{l}\text { Es una entidad continua que depende de uno u otros continuos independientes que le sirven como } \\
\text { portadores, por ejemplo, las entidades de información creadas por los seres humanos }\end{array}$ \\
\hline Entidad que ocurre & $\begin{array}{l}\text { Es una entidad que sucede, se despliega o desarrolla en el tiempo } \\
\text { Es una entidad que se desenvuelve a sí misma en el tiempo o es la frontera instantánea de dicha entidad, } \\
\text { por ejemplo, un inicio o un final }\end{array}$ \\
\hline Proceso & $\begin{array}{l}\text { Es una entidad que ocurre, que existe en un tiempo, tiene partes temporales y siempre depende de al } \\
\text { menos una entidad material }\end{array}$ \\
\hline Historia & $\begin{array}{l}\text { Esta entidad representa la suma de todos los procesos que tienen lugar en una región espaciotemporal } \\
\text { ocupada por la entidad material o el sitio en cuestión }\end{array}$ \\
\hline Perfil del proceso & $\begin{array}{l}\text { Es una entidad que se posiciona en un nivel de complejidad algo más elevado } \\
\text { Se enfoca no en determinadas magnitudes de calidad trazadas a lo largo del tiempo, sino más bien en } \\
\text { ciertas relaciones entre estas magnitudes y los tiempos transcurridos con el fin de obtener una ratio que } \\
\text { caracterice el proceso }\end{array}$ \\
\hline Límite del proceso & $\begin{array}{l}\text { Es el límite temporal de un proceso en un instante dado } \\
\text { Es el comienzo y el final del proceso que este limita } \\
\text { El límite del proceso es una parte temporal que en sí misma no tiene partes temporales porque ella } \\
\text { misma es la mínima parte temporal del proceso }\end{array}$ \\
\hline Región temporal & $\begin{array}{l}\text { Es una parte del tiempo total, la región temporal a diferencia de la región espaciotemporal solo se } \\
\text { extiende y limita en la dimensión temporal } \\
\text { Dado que no existe el tiempo absoluto requiere para su representación, como la espacial, la selección de } \\
\text { un marco de referencia }\end{array}$ \\
\hline Región espaciotemporal & $\begin{array}{l}\text { Es una entidad en la que pueden localizarse entidades que ocurren } \\
\text { Es una parte del espacio/tiempo total } \\
\text { Cada región espaciotemporal se define con relación a un marco de referencia que involucra un sistema } \\
\text { de coordenadas de cuatro dimensiones }\end{array}$ \\
\hline
\end{tabular}

Tabla 4. Clases incluídas en BFO

Fuente de los datos: Arp y Smith (2008, 2011); Arp, Smith y Spear (2015)

De acuerdo con Arp, Smith y Spear (2015), es fundamental comprender que las ontologías intentan sobre todo representar a los universales y a las relaciones existentes entre ellos. Las afirmaciones sobre tales relaciones constituyen la mayor parte del conocimiento científico.

\section{Relaciones y axiomas en BFO}

$\mathrm{BFO}$, como ontología de nivel superior, reconoce tres tipos de relaciones binarias (Tabla 5):

»Relaciones entre dos universales, es típica la relación is_a entre un universal más específico "hijo", y uno más general "padre" debido a que los universales se organizan jerárquicamente

» relaciones entre un universal y un particular, la más común es la relación de instanciación

» relaciones entre dos particulares, por ejemplo, part_of 
Tabla 5. Relaciones binarias

\begin{tabular}{ll}
\hline \multicolumn{1}{c}{ Relaciones binarias } & \multicolumn{1}{c}{ Ejemplos } \\
\hline $\begin{array}{l}\text { Entre dos universales } \\
<\text { clase, clase }\end{array}$ & $\begin{array}{l}\text { Is_a entre un universal más } \\
\text { específico y un universal más } \\
\text { general }\end{array}$ \\
\hline $\begin{array}{l}\text { Entre un particulary } \\
\text { un universal } \\
<\text { instancia, clase> }\end{array}$ & Instanciación, instance_of \\
\hline $\begin{array}{l}\text { Entre dos particulares } \\
<\text { instancia, instancia> }\end{array}$ & part_of \\
\hline
\end{tabular}

Fuente de los datos: Smith et al. (2005), Arp, Smith y Spear (2015)

Por lo tanto, para representar y capturar la información científica relevante en un dominio dado, se requiere considerar y definir las relaciones existentes entre los universales, así como enunciar axiomas sobre ellas. La relación is_a es central al diseñar la arquitectura de la ontología para garantizar el principio de herencia única que sustentan las definiciones aristotélicas. Al definir cada relación es importante especificar las categorías de objetos a los que puede asignárseles un dominio y un rango en dicha relación. Dado que las relaciones se comportan de manera diferente según se trate de entidades continuas y entidades que ocurren, se distinguen las relaciones partitivas de cada una. Asimismo, se han establecido convenciones para representar las relaciones de universales y particulares (Smith et al., 2005; Arp, Smith y Spear, 2015):

» Las variables en letra mayúscula C, D, .... se usan para representar universales continuos

» Las variables en letra minúscula $c, d, \ldots$ se usan para representar particulares continuos

» Las variables en letra mayúscula $P, Q_{2} \ldots$ se usan para representar universales que ocurren

» Las variables en letra minúscula $p, q, \ldots$ se usan para representar particulares que ocurren

» La relación entre dos universales se representa en itálica, por ejemplo: $C$ is_a $\mathrm{D}$; $\mathrm{P}$ is_a $\mathrm{Q}$

"La relación entre un particular y un universal se representa en negrita, por ejemplo: $c$ instance_of $C$; $p$ instance_of $P$

"La relación entre dos particulares, también se escribe en negrita: c continuant_ part_of $\mathrm{c}$ at $\mathrm{t}$; $\mathrm{p}$ ocurrent_part_of $\mathrm{q}$

La aplicación de estas convenciones a las tres familias de relaciones se plasma, a continuación, en los siguientes ejemplos (Tabla 6):

\begin{tabular}{lll}
\hline Tipo de Relación & Tipo de entidad & Ejemplo \\
\hline universal-universal & $\begin{array}{l}\text { entidades continuas } \\
\text { entidades que ocurren }\end{array}$ & $\begin{array}{l}\text { objecto is_a continuo independiente } \\
\text { proceso is_a entidad que ocurre }\end{array}$ \\
\hline particular-universal & $\begin{array}{l}\text { entidades continuas } \\
\text { entidades que ocurren }\end{array}$ & $\begin{array}{l}\text { este autor instance_of autor } \\
\text { esta interpretación instance_of } \\
\text { interpretación }\end{array}$ \\
\hline particular-particular & $\begin{array}{l}\text { entidades continuas } \\
\text { entidades que ocurren }\end{array}$ & $\begin{array}{l}\text { este capítulo de libro continuant_part_of } \\
\text { este libro } \\
\text { la interpretación de esta escena } \\
\text { continuant_part_of este proceso de } \\
\text { interpretación de esta obra de teatro }\end{array}$ \\
\hline
\end{tabular}

Tabla 6. Ejemplificación de las convenciones BFO para la representación de relaciones 
Existen también convenciones para enunciar las afirmaciones que hacen referencia a las variables de tiempo y espacio:

»Variables $r, r$, ... para representar regiones espaciales tridimensionales

"Variables $t, t$ ', ... para representar instancias de tiempo

Estas variables intervienen en la definición de las relaciones primitivas (Smith, et al., 2005) (Tabla 7). Su significado se dilucida informalmente a partir de los ejemplos y se formaliza mediante el enunciado de axiomas:

\begin{tabular}{|c|c|}
\hline \multicolumn{2}{|r|}{ Relaciones Primitivas } \\
\hline Relación & Definición \\
\hline c instance_of $\mathrm{C}$ at $\mathrm{t}$ & $\begin{array}{l}\text { Relación primitiva dada en un tiempo específico entre una instancia } \\
\text { continua c y un universal continuo C, cuando el primero, instancia } \\
\text { al segundo en dicho tiempo }\end{array}$ \\
\hline p instance_of $P$ & $\begin{array}{l}\text { Relación primitiva entre la instancia de un proceso y el universal } \\
\text { correspondiente de ese proceso }\end{array}$ \\
\hline c continuant_part_of d at $t$ & $\begin{array}{l}\text { Relación primitiva entre dos instancias continuas en un tiempo } \\
\text { dado, donde la primera es parte de la otra }\end{array}$ \\
\hline p occurrent_part_of q & $\begin{array}{l}\text { Relación primitiva partitiva entre instancias de un proceso cuando } \\
\text { una es un subproceso de la otra. }\end{array}$ \\
\hline r continuant_part_of r' & $\begin{array}{l}\text { Relación primitiva partitiva entre regiones espaciales (una, } \\
\text { subregión de la otra) }\end{array}$ \\
\hline c inheres_in $\mathrm{d}$ at $\mathrm{t}$ & $\begin{array}{l}\text { Relación primitiva entre una entidad continua específicamente } \\
\text { dependiente y una entidad continua independiente en un tiempo } \\
\text { dado }\end{array}$ \\
\hline c located_in $r$ at t & $\begin{array}{l}\text { Relación primitiva entre una instancia continua y la región espacial } \\
\text { que ella ocupa en un tiempo dado }\end{array}$ \\
\hline r adjacent_to r' & Relación primitiva de proximidad entre dos regiones espaciales \\
\hline c derives_from d & $\begin{array}{l}\text { Relación primitiva entre dos entidades continuas materiales } \\
\text { diferentes cuando una sucede a la otra y deriva de ella }\end{array}$ \\
\hline p has_participant c & Relación primitiva entre un proceso y una entidad continua \\
\hline
\end{tabular}

Fuente de los datos: Smith et al. (2005); Arp, Smith y Spear (2015)

Las relaciones se han clasificado y agrupado en cuatro categorías (Tabla 8):

\begin{tabular}{|l|l|}
\hline \multicolumn{1}{|c|}{ Categoría } & \multicolumn{1}{c|}{ Relación } \\
\hline Relación fundacional & $\begin{array}{l}\text { Relación primitiva dada en un tiempo específico entre una instancia } \\
\text { continua c y un universal continuo C, cuando el primero, instancia al } \\
\text { segundo en dicho tiempo }\end{array}$ \\
\hline Relación espacial & $\begin{array}{l}\text { Relación primitiva entre la instancia de un proceso y el universal } \\
\text { correspondiente de ese proceso }\end{array}$ \\
\hline Relación temporal & $\begin{array}{l}\text { Relación primitiva entre dos instancias continuas en un tiempo dado, } \\
\text { donde la primera es parte de la otra }\end{array}$ \\
\hline $\begin{array}{l}\text { Relación de } \\
\text { participación }\end{array}$ & $\begin{array}{l}\text { Relación primitiva partitiva entre instancias de un proceso cuando una es } \\
\text { un subproceso de la otra }\end{array}$ \\
\hline
\end{tabular}

Fuente de los datos: Smith et al. (2005); Arp, Smith y Spear (2015)
Tabla 7. Relaciones primitivas

Tabla 8. Clasificación de las relaciones 
Así las relaciones se categorizan y se definen formalmente de la siguiente manera (Tabla 9):

\begin{tabular}{|c|c|c|}
\hline \multicolumn{3}{|r|}{ Relaciones básicas } \\
\hline Categorías & Relaciones & Definiciones y propiedades \\
\hline \multirow[t]{3}{*}{$\begin{array}{l}\text { Relaciones } \\
\text { fundacionales }\end{array}$} & is_a & $\begin{array}{l}\mathrm{A} \text { is_a } \mathrm{B}=\operatorname{def} . \mathrm{A} \text { y } \mathrm{B} \text { son universales y para todo } \mathrm{x} \\
\text { Propiedades transitiva, reflexiva, antisimétrica. }\end{array}$ \\
\hline & continuant_part_of & $\begin{array}{l}\text { C continuant_part_of } \mathrm{D}=\text { def. para todo particular continuo } \mathrm{c} y \text { todo } \\
\text { tiempo } t \text {, si c instance_of } C \text { at } t \text {, entonces hay algún } d \text { instance_of } D \text { at } t y \\
\text { c es un continuant_part_of } d \text { at } t \\
\text { Propiedades transitiva, reflexiva, antisimétrica }\end{array}$ \\
\hline & occurrent_part_of & $\begin{array}{l}\text { P occurrent_part_of } Q=\text { def. para todo particular que ocurre } p \text {, si } p \\
\text { instance_of } P \text {, entonces hay algún particular que ocurre } q \text { tal que } q \\
\text { instance_of } Q y \text { p occurrent_part_of } q \\
\text { Propiedades transitiva, reflexiva, antisimétrica }\end{array}$ \\
\hline \multirow[t]{2}{*}{$\begin{array}{l}\text { Relaciones } \\
\text { espaciales }\end{array}$} & located_in & $\begin{array}{l}\text { c located_in } \mathrm{d} \text { at } \mathrm{t}=\text { def. hay dos regiones espaciales } \mathrm{r} \text { y r’ tal que el } \\
\text { particular continuo c está located_in } r \text { at } \mathrm{t} \text { y el particular continuo d está } \\
\text { located_in r' at t, y la región } r \text { es una continuant_part_of la región r' } \\
\text { Propiedades transitiva, reflexiva }\end{array}$ \\
\hline & adjacent_to & $\begin{array}{l}\text { C adjacent_to } D=\text { def. Para todo particular continuo c y para todo tiempo } \\
t \text {, si c instance_of } C \text { at } t \text {, entonces hay algún } d \text { tal que } d \text { instance_of } D \text { at } t \\
\text { y c adjacent_to } d \text { at } t \\
\text { Propiedad simétrica }\end{array}$ \\
\hline \multirow[t]{2}{*}{$\begin{array}{l}\text { Relaciones } \\
\text { temporales }\end{array}$} & derives_from & $\begin{array}{l}C \text { derives_from } \mathrm{D}=\text { def. para todo particular continuo c } \mathrm{y} \text { todo tiempo } t \text {, } \\
\text { si } c \text { instance_of } C \text { at } t \text {, entonces hay algún } d y \text { algún tiempo anterior } t^{\prime} \text { tal } \\
\text { que } d \text { instance_of } D \text { at } t^{\prime} \text {, and } c \text { derives_from } d\end{array}$ \\
\hline & preceded_by & $\begin{array}{l}\text { P preceded_by } Q=\text { def. para todo particular que ocurre } p \text {, si } p \text { instance_of } \\
P \text {, entonces hay algún } q \text {, tal que } q \text { instance_of } Q \text { y p preceded_by } q \\
\text { Propiedad transitiva }\end{array}$ \\
\hline $\begin{array}{l}\text { Relaciones de } \\
\text { participación }\end{array}$ & has_participant & $\begin{array}{l}\text { P has_participant } C=\text { def. para todo particular que ocurre } p \text {, si } p \text { instance } \\
\text { of } P \text {, entonces hay algún } c y \text { algún tiempo } t \text { tal que } c \text { instance_of } C \text { at } t \text { y } p \\
\text { has_participant } c \text { at } t\end{array}$ \\
\hline
\end{tabular}

Tabla 9. Relaciones básicas.

Fuente de los datos: Smith et al. (2005); Arp, Smith y Spear (2015)

Dichas relaciones no admiten excepciones. Ello se refleja en la regla Todas-Alguna (All-Some rule) que indica para todo universal A portador de una relación con un universal B, que todas las instancias relevantes de A deben ser portadoras de dicha relación, en el nivel de las instancias, de alguna instancia de B. Además, tal como se puntualiza en el cuadro (Tabla 9), algunas relaciones, en el nivel de las instancias, poseen propiedades (reflexividad, simetría, transitividad, asimetría). Una relación $\mathrm{R}$ es reflexiva cuando cualquier $\mathrm{A}$, portador de la relación $\mathrm{R}$ con algo más, $\mathrm{B}$, porta también dicha relación con respecto a sí mismo. Es simétrica si $\mathrm{A}$ está en $\mathrm{R}$ con respecto a $\mathrm{B}$ y $\mathrm{B}$ también se encuentra en $\mathrm{R}$ con respecto a $\mathrm{A}$ (por ejemplo, si A está cerca de B, B está cerca de A); en el nivel de las instancias la relación adjacent_to es simétrica. Una relación $\mathrm{R}$ es transitiva si A es portador de 
$\mathrm{R}$ con respecto a $\mathrm{B}, \mathrm{y} \mathrm{B}$ es portador de $\mathrm{R}$ con respecto a $\mathrm{C}$, entonces $\mathrm{A}$ es portador de $R$ con respecto a $C$. Una relación es antisimétrica si A es portador de $R$ con respecto a B y B es portador de $\mathrm{R}$ con respecto a A, entonces A y B son idénticos (Arp, Smith y Spear, 2015).

Se han definido, también, otras relaciones de importancia, de nivel superior, tales como las existentes entre el todo y la/s parte/s (Tabla 10):

\begin{tabular}{|c|c|}
\hline \multicolumn{2}{|r|}{ Otras relaciones de nivel superior } \\
\hline Relación & Definición \\
\hline $\begin{array}{l}\text { Proper_continuant_ } \\
\text { part_of }\end{array}$ & $\begin{array}{l}\text { C proper_continuant_part_of } \mathrm{D}=\text { def. para todo particular continuo c } \\
\text { y todo tiempo t, si c instance_of C at t, entonces hay algún d tal que d } \\
\text { instance_of D at t, y c proper_continuant_part_of d at t } \\
\text { Hablar de la parte apropiada, correcta de algo (“proper part of') en el nivel } \\
\text { de las instancias, es referirse a algo que es parte, aunque NO idéntica de } \\
\text { algo más }\end{array}$ \\
\hline $\begin{array}{l}\text { proper_occurrent_ } \\
\text { part_of }\end{array}$ & $\begin{array}{l}\text { P proper_occurrent_part_of } Q=\text { def. para todo particular que ocurre p, si p } \\
\text { instance_of } P \text {, entonces hay algún particular q tal que q instance_of } Q, p \\
\text { proper_occurrent_part_of } q\end{array}$ \\
\hline has continuant_part & $\begin{array}{l}\text { C has continuant_part } D=\text { def. para todo particular continuo } c, y \text { todo } \\
\text { tiempo } t \text {, si c instances_of } C \text { at } t \text { entonces hay algún } D \text {, tal que } d \text { instance_ } \\
\text { of } D \text { at } t \text {, } y \text { d continuant_part_of } c \text { at } t \\
\text { Afirmar de dos universales que el primero tiene al segundo como parte, } \\
\text { implica enunciar que el primero tiene instancias que constituyen un todo, } \\
\text { y que cada una de estas tiene una instancia del segundo como parte }\end{array}$ \\
\hline has_occurrent_part & $\begin{array}{l}P \text { has_occurrent_part } Q=\text { def. para todo particular que ocurre } p, \text { si } \\
\text { p instance_of } P \text { entonces hay algún } q \text {, tal que } q \text { instance_of } Q \text {, y } q \\
\text { occurrent_part_of } p\end{array}$ \\
\hline part_of/ has_part & $\begin{array}{l}\text { Hay una relación de "parte integral” (“integral parthood”) entre dos } \\
\text { universales A y B cuando A es part_of B y B has_part A: } \\
\text { C integral_continuant_part_ofD y D has_continuant_part C } \\
\text { P integral_occurrent_part_of Q y Q has_occurrent_part P }\end{array}$ \\
\hline
\end{tabular}

Fuente de los datos: Arp, Smith y Spear (2015)
Tabla 10. Otras relaciones de nivel superior

Por último, cabe considerar las relaciones de inversión y reciprocidad (Arp, Smith y Spear, 2015). La inversa de una relación $\mathrm{R}$ se define como la relación que se obtiene entre cada par de entidades relacionadas mediante $\mathrm{R}$ cuando se tiene en cuenta en reversa dicha relación. De manera que, si $\mathrm{C}$ is_a $\mathrm{D}$, la relación entre $\mathrm{D}$ y $\mathrm{C}$ que se orienta en la dirección opuesta es la relación has_subuniversal, definida como $\mathrm{C}$ has_subuniversal $\mathrm{D}=$ def. $\mathrm{D}$ is_a $\mathrm{C}$, como en documento has_subuniversal artículo de revista. Este tipo de relación permite el desplazamiento vertical a través de la taxonomía de una ontología sobre la base del principio de la herencia.

En la mayoría de los casos, no es posible definir en forma directa una relación inversa entre entidades, por ello es necesario definir relaciones reciprocas. Arp, Smith y Spear (2015), advierten que la relación has_continuant_part no es la inversa sino la recíproca de continuant_part_of.; tal como sucede con la relación adjacent_to.

Una vez definidas las relaciones, es posible enunciar axiomas que las operativicen. Algunos axiomas básicos en BFO son (Tabla 11): 


\begin{tabular}{|c|c|}
\hline \multicolumn{2}{|r|}{ Axiomas } \\
\hline Entidad & $\begin{array}{l}\text { Todas las entidades existen en alguna región temporal } \\
\forall \mathrm{x}(\text { Entity }(\mathrm{x}) \rightarrow \exists \mathrm{t}(\text { TemporalRegion }(\mathrm{t}) \Lambda \text { exists_at }(\mathrm{x}, \mathrm{t})))\end{array}$ \\
\hline Entidad material & $\begin{array}{l}\text { Toda entidad material tiene una historia. } \\
\forall \mathrm{x}(\text { MaterialEntity }(\mathrm{x}) \rightarrow \exists \mathrm{y} \text { has_history }(\mathrm{x}, \mathrm{y})) \\
\text { Toda entidad material existe en algún intervalo temporal } \\
\forall \mathrm{x}(\text { MaterialEntity }(\mathrm{x}) \rightarrow \exists \mathrm{t}(\text { 1DTemporalRegion }(\mathrm{t}) \Lambda \text { exists_at }(\mathrm{x}, \mathrm{t})))\end{array}$ \\
\hline Entidad que ocurre & $\begin{array}{l}\text { Toda entidad que ocurre ocupa alguna región espaciotemporal } \\
\forall \mathrm{x}(\text { Occurrent }(\mathrm{x}) \rightarrow \exists \mathrm{y}(\text { SpatioTemporalRegion }(\mathrm{y}) \Lambda \text { occupies } \\
\text { spatiotemporal_region }(\mathrm{x}, \mathrm{y})))\end{array}$ \\
\hline $\begin{array}{l}\text { Región } \\
\text { espaciotemporal }\end{array}$ & $\begin{array}{l}\text { Toda región espaciotemporal ocupa alguna región temporal } \\
\forall \mathrm{x}(\text { SpatioTemporalRegion }(\mathrm{x}) \rightarrow \exists \mathrm{t}(\text { TemporalRegion }(\mathrm{t}) \Lambda \text { occupies__ } \\
\text { temporal_region }(\mathrm{x}, \mathrm{t}))) \\
\text { Toda región espaciotemporal } \text { es ocupada por sí misma } \\
\forall \mathrm{x}(\text { SpatioTemporalRegion }(\mathrm{x}) \rightarrow \text { occupies_spatiotemporal_region }(\mathrm{x}, \mathrm{x}))\end{array}$ \\
\hline Región temporal & $\begin{array}{l}\text { Todas las regiones temporales son cero- o unidimensionales (instantes o } \\
\text { intervalos) } \\
\forall \mathrm{x}(\text { TemporalRegion }(\mathrm{x}) \leftrightarrow(1 \mathrm{DT} \text { TemporalRegion }(\mathrm{x}) \Lambda \\
\text { 0DTemporalRegion }(\mathrm{x}))) \\
\text { Toda región temporal } \text { es ocupada por sí misma } \\
\forall \mathrm{x}(\text { TemporalRegion }(\mathrm{x}) \rightarrow \text { occupies_temporal_region }(\mathrm{x}, \mathrm{x}))\end{array}$ \\
\hline Universal & $\begin{array}{l}\text { Algo es un universal si y solo si es instanciado por algo } \\
\forall \mathrm{X} \text { (Universal(X) } \leftrightarrow \exists \mathrm{y} \text { inst }(\mathrm{X}, \mathrm{y}))\end{array}$ \\
\hline $\begin{array}{l}\text { Es parte de una } \\
\text { entidad continua }\end{array}$ & 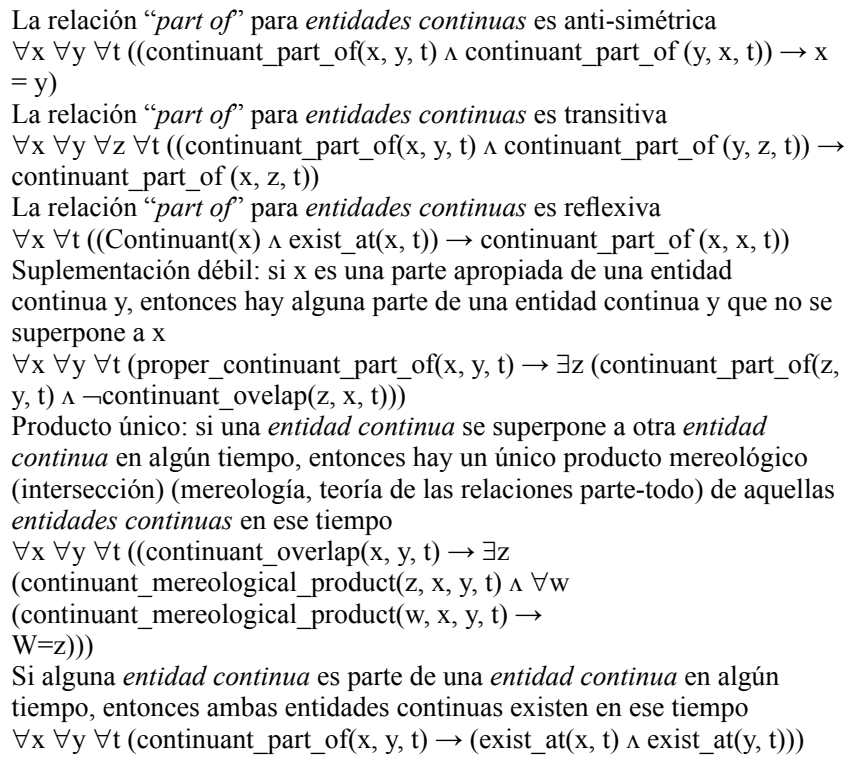 \\
\hline $\begin{array}{l}\text { Ocupa una región } \\
\text { espacial }\end{array}$ & $\begin{array}{l}\text { Algo solo puede ocupar una única región espacial } \text { en un mismo tiempo } \\
\forall \mathrm{x} \forall \mathrm{r}_{1} \forall \mathrm{r}_{2} \forall \mathrm{t} \text { (occupies_spatial_region }\left(\mathrm{x}, \mathrm{r}_{1}, \mathrm{t}\right) \Lambda \text { occupies_spatial_ } \\
\left.\text { region }\left(\mathrm{x}, \mathrm{r}_{2}, \mathrm{t}\right) \rightarrow \mathrm{r}_{1}=\mathrm{r}_{2}\right) \\
\text { Todas las entidades que ocupan una región espacial } \text { en un mismo tiempo } \\
\text { existen en ese tiempo } \\
\forall \mathrm{x} \forall \mathrm{r} \forall \mathrm{t} \text { (occupies_spatial_region }(\mathrm{x}, \mathrm{r}, \mathrm{t}) \rightarrow \text { exist_at }(\mathrm{x}, \mathrm{t}))\end{array}$ \\
\hline $\begin{array}{l}\text { Ocupa una región } \\
\text { espaciotemporal }\end{array}$ & $\begin{array}{l}\text { Algo solo puede ocupar una única región espaciotemporal } \\
\forall \mathrm{x} \forall \mathrm{r}_{1} \forall \mathrm{r}_{2}\left(\left(\text { occupies_spatiotemporal_region }\left(\mathrm{x}, \mathrm{r}_{1}\right) \Lambda \text { occupies_ }\right.\right. \\
\left.\left.\text { spatiotemporal_region }\left(\mathrm{x}, \mathrm{r}_{2}\right)\right) \rightarrow \mathrm{r}_{1}=\mathrm{r}_{2}\right)\end{array}$ \\
\hline
\end{tabular}

En la optimización de la capacidad de generar inferencias automáticamente, influyen, además de los axiomas, la expresividad de los lenguajes utilizados al diseñar las ontologías. RDF Schema (RDFS) introduce relaciones que le otorgan expresividad, como la propiedad authored_by con dominio "documento" y rango "persona", pero no reconoce, por ejemplo, la propiedad transitiva, la reflexiva o la simétrica. Estas y otras limitaciones llevaron a la creación de OWL, que permite la expresión de: 
1. Cuantificación universal $(\forall)$, a través de la restricción owl:allValuesFrom

2. Cuantificación existencial $(\exists)$, a través de la restricción owl:someValuesFrom u owl:hasValue

3. Cardinalidad, owl:cardinality, owl:minCardinality, y owl:maxCardinality

4. Operadores booleanos and, or, y not, owlintersectionOf, owl:unionOf, y owl:complementOf

5. Afirmación de equivalencia, owl:equivalentClass y owl:equivalentProperty

6. Propiedades:

a) Inversa (owl:inverseOf)

b) Funcional (owl:FunctionalProperty)

c) Función inversa (owl:InverseFunctionalProperty)

d) Transitiva (owl:TransitiveProperty)

e) Simétrica (owl:SymmetricProperty)

f) Asimétrica (owl:AsymmetricProperty)

g) Reflexiva (owl:ReflexiveProperty)

h) Irreflexiva (owl:IrreflexiveProperty)

OWL, se ha basado en el estándar Description Logics (DLs). Este estándar, tiene aún limitaciones desde el punto de vista lógico que pueden superarse a futuro, pero posee propiedades computacionales ventajosas para el razonamiento y la validación de datos en las ontologías (Arp, Smith y Spear, 2015). Dado el amplio uso de este lenguaje, se ha publicado ya una versión actualizada de la ontología en OWL, la BFO 2.0 OWL.

\section{Conclusión}

En síntesis, una buena ontología material, en primer lugar, se piensa. Requiere de un proceso de pensamiento que dé respuesta a la necesidad que justifica su diseño y permita seleccionar los términos adecuados, en concordancia con el contexto científico que constituye el marco del dominio a representar. Pero una vez bosquejada esta instancia, es preciso darle forma como artefacto que pueda gestionarse a través de una computadora. En esa etapa, la posibilidad de aumentar la capacidad de inferencia automática de este artefacto depende de la expresividad del lenguaje utilizado y de la creación de definiciones formales que den lugar al enunciado de axiomas. Por ese motivo, resulta fundamental dar el tratamiento adecuado al nivel lógico de la ontología. 


\section{Referencias bibliográficas}

》Arp, Robert y Barry Smith. 2008. Function, Role, and Disposition in Basic Formal Ontology [preprint]. <http://precedings.nature.com/documents/1941/version/1> [Consulta: 19 enero 2018].

» Arp, Robert y Barry Smith. 2011. Realizable Entities in Basic Formal Ontology (revised version). <http://ontology.buffalo.edu/smith/articles/realizables.pdf> [Consulta: 15 enero 2018].

»Arp, Robert y Barry Smith y Andrew D. Spear. 2015. Building Ontologies with Basic Formal Ontology. Cambridge, MA: The MIT Press.

»Gruber, Thomas R. 1993. A translation approach to portable ontology specifications. En Knowledge Acquisition. Vol. 5, no. 2, 199-220. < http://tomgruber.org/ writing/ontolingua-kaj-1993.pdf> [Consulta: 15 enero 2018].

»Guarino, Nicola. 1995. Formal ontology, conceptual analysis and knowledge representation. En International Journal of Human-Computer Studies. Vol. 43, nos., 5-6, 625-640. <https://www.sciencedirect.com/journal/international-journal-of-human-computer-studies/vol/43/issue/5> [Consulta: 15 enero 2018].

» Guarino, Nicola. 1998. Formal Ontology and Information Systems. Versión modificada del artículo publicado en Guarino, N., ed. Formal Ontology in Information Systems. Proceedings of FOIS'98 (6-8 June 1998: Trento, Italy). Amsterdam: IOS Press, p. 3-15. <https://www.academia.edu/516497/Formal_ontology_in_information_systems $>$ [Consulta: 9 enero 2018].

" Guarino, Nicola y Christopher Welty. 2000. A Formal Ontology of Properties. En Dieng, Rosey Olivier Corby, eds. Knowledge Engineering and KnowledgeManagement:Methods, Models, and Tools: 12th International Conference, EKAW 200o. Proceedings (2-6 October 2000: Juan-les-Pins, France). Berlin: Springer, p. 97-112. <https:// archive.org/stream/springer_10.1007-3-540-39967-4/10.1007-3-540-39967-4_djvu. txt> [Consulta: 9 enero 2018].

"Guarino, Nicola y Christopher Welty. 2009. An Overview of OntoClean. En Staab, S. y Studer, R. eds. Handbook on ontologies. Berlin: Springer, p. 201-220. $<$ https://www.researchgate.net/publication/226934944_An_Overview_of_OntoClean> [Consulta: 3 febrero 2018].

" Guarino, Nicola, Daniel Oberle y Steffen Staab. 2009. What is an Ontology? En Staab, Steffen. y Studer, R. eds. Handbook on ontologies. Berlin: Springer, p. 1-17. <http://www.iaoa.org/isc2012/docs/Guarino2009_What_is_an_Ontology.pdf> [Consulta: 3 febrero 2018].

» Mascardi, Viviana., Valentina Cordì y Paolo Rosso. 2007. A Comparison of Upper Ontologies. <https://www.researchgate.net/publication/220866366_A_Comparison_of_Upper_Ontologies> [Consulta: 3 febrero 2018].

"Poli, Roberto, Michael Healy y Achilles. D. Kameas. eds. 2010. Theory and Applications of Ontology: Computer Applications, Berlin: Springer Science+Business Media B.V. <http://marte.aslab.upm.es/redmine/files/dmsf/p_ oasys/160623124554_195_Poli_-_Theory_and_Applications_of_Ontology.pdf> [Consulta: 15 de noviembre de 2018].

"Shaw, Marianne, Landon T. Detwiler, James M. Brinkley y Dan Suciu. 2008. Generating Application Ontologies from Reference Ontologies. En AMIA 2008 
Symposium Proceedings. Bethesda, Maryland, p. 672-676. <http://citeseerx.ist. psu.edu/viewdoc/download?doi=10.1.1.500.4289\&rep=rep1\&type=pdf> [Consulta: 9 enero 2018].

»Smith, Barry. 1998. Basic Concepts of Formal Ontology. En Guarino, Nicola, ed. Formal Ontology in Information Systems. Amsterdam: IOS Press, p. 19-28. <https://philarchive.org/archive/SMITBT> [Consulta: 30 agosto 2016].

"Smith, Barry. 2015. Basic Formal Ontology 2.0: Specification and user's guide. $<$ https://raw.githubusercontent.com/BFO-ontology/BFO/v2.o/BFO2-Reference.docx> [Consulta: 3 febrero 2018].

»Smith, Barry, Werner Ceusters, Bert Klagges, Jakob Köhler, Anand Kumar, Jane Lomax, Chris Mungall, Fabian Neuhaus, Alan L. Rector y Cornelius Rosse. 2005. Relations in Biomedical Ontologies. En Genome Biology. Vol. 6, no. 5 R46, 1-15. <https://doi.org/10.1186/gb-2005-6-5-r46> [Consulta: 7 febrero 2018].

»Smith, Barry, Waclaw Kusnierczyk, Daniel Schober y Werner Ceusters. 2006. Towards a Reference Terminology for Ontology Research and Development in the Biomedical Domain. En Bodenreider, O., ed. Proceedings of the 2nd International Workshop on Formal Biomedical Knowledge Representation (2nd; 2006). Trabajos presentados. Baltimore, MD: KR-MED Publications. Vol. 222, p. 57-66. <http:// citeseerx.ist.psu.edu/viewdoc/download?doi=10.1.1.142.9278\&rep=rep1\&type $=$ pdf $>$ [Consulta: 10 agosto 2016].

»Vitturini, Mercedes y Pablo Rubén Fillottrani. 2012. Ontologies Models Cohesiveness: A First Assessment of Integration. En Journal of Computer Science $\varepsilon$ Technology. Vol. 12, no. 3, 104-109. <http://hdl.handle.net/10915/22053> [Consulta: 5 enero 2018].

»Wache, Holger, Thomas Vögele, Ubbo Visser, Heiner Stuckenschmidt, G. Schuster, H. Neumann y Sebastian Hübner. 2001. Ontology-based Integration of Information - A Survey of Existing Approaches, En Proceedings of IJCAl-o1 Workshop: Ontologies and Information Sharing. Seattle, WA, p. 108-117. <http:// citeseerx.ist.psu.edu/viewdoc/download?doi=10.1.1.142.439o\&rep=rep1\&type $=p d f>$ [Consulta: 5 enero 2018]. 
